\title{
Fuel level sensor based on polymer optical fiber Bragg gratings for aircraft applications
}

\author{
C. A. F. Marques ${ }^{a^{*}}$, A. Pospori ${ }^{\mathrm{a}}$, D. Sáez-Rodríguez ${ }^{\mathrm{b}}$, K. Nielsen ${ }^{\mathrm{c}}$, O. Bang ${ }^{\mathrm{c}}$, D. J. Webb ${ }^{\mathrm{a}}$ \\ ${ }^{a}$ Aston Institute of Photonic Technologies, Aston University, B4 7ET, Birmingham, UK \\ ${ }^{b}$ Comunicaciones Opticas, Universidad Politecnica de Valencia, Valencia 46022, Spain \\ 'DTU Fotonik, Department of Photonics Engineering, DK-2800 Kgs. Lyngby, Denmark \\ *E-mail contact: c.marques@aston.ac.uk
}

\begin{abstract}
Safety in civil aviation is increasingly important due to the increase in flight routes and their more challenging nature. Like other important systems in aircraft, fuel level monitoring is always a technical challenge. The most frequently used level sensors in aircraft fuel systems are based on capacitive, ultrasonic and electric techniques, however they suffer from intrinsic safety concerns in explosive environments combined with issues relating to reliability and maintainability. In the last few years, optical fiber liquid level sensors (OFLLSs) have been reported to be safe and reliable and present many advantages for aircraft fuel measurement. Different OFLLSs have been developed, such as the pressure type, float type, optical radar type, TIR type and side-leaking type. Amongst these, many types of OFLLSs based on fiber gratings have been demonstrated. However, these sensors have not been commercialized because they exhibit some drawbacks: low sensitivity, limited range, long-term instability, or limited resolution. In addition, any sensors that involve direct interaction of the optical field with the fuel (either by launching light into the fuel tank or via the evanescent field of a fiber-guided mode) must be able to cope with the potential build up of contamination - often bacterial - on the optical surface. In this paper, a fuel level sensor based on microstructured polymer optical fiber Bragg gratings (mPOFBGs), including poly (methyl methacrylate) (PMMA) and TOPAS fibers, embedded in diaphragms is investigated in detail. The mPOFBGs are embedded in two different types of diaphragms and their performance is investigated with aviation fuel for the first time, in contrast to our previous works, where water was used. Our new system exhibits a high performance when compared with other previously published in the literature, making it a potentially useful tool for aircraft fuel monitoring.
\end{abstract}

Keywords: Polymer optical fiber sensors, Bragg gratings, fuel level sensors, diaphragms, aircraft applications.

\section{INTRODUCTION}

Polymer optical fibers (POFs) are starting to be considered a viable alternative to silica fibers in applications such as short distance transmissions [1], Terahertz waveguides and filters [2], and mainly in sensing applications [3,4], due to their flexibility, large cores, high failure strain and great elasticity. The mechanical properties provide enhanced sensitivity or longer operational range to intrinsic polymer fibre sensors when they are used for strain [5,6], stress [7], pressure [8], temperature [9,10] and humidity [11] monitoring, as well as for transverse force sensing [12]. Many POF sensors are based on fibre Bragg gratings (FBGs), which have been written in different spectral windows in doped and undoped step-index POFs [13], microstructured fibers (including PMMA and TOPAS material) [13-15], as well as low loss cyclic transparent optical polymer (CYTOP)-perfluorinated POFs [16], and graded-index POFs [17]. Polymer optical fiber Bragg gratings (POFBGs) are inscribed with different laser systems including continuous-wave (CW) $\mathrm{HeCd}$ laser (@325 nm) [13-15], pulsed KrF laser (@248 nm) [18], and also femtosecond laser systems [16].

In recent years, optical fiber liquid level sensors (OFLLSs) have been reported to be safe and reliable and present many advantages for aircraft fuel measurement [19]. Different OFLLSs have been developed, such as the pressure type, float type, optical radar type, total internal reflection type and side-leaking type. Amongst these, many types of OFLLSs based on fiber gratings have been demonstrated [20-23]. However, these sensors have not been commercialized because they exhibit some drawbacks: low sensitivity, limited range, long-term instability, or limited resolution.

In this paper, it is shown that mPOFBGs embedded in diaphragms can be useful to monitor the level of fuel, in this case JET A-1 fuel from aircraft. Five diaphragm-based pressure sensors were used, which are monitored using a linear array of POFBGs. Recently, we reported the study of these sensors using water, revealing an average sensitivity of $98.2 \pm 0.4$ 
$\mathrm{pm} / \mathrm{cm}$ water depth. Furthermore, initial testing in JET A-1 aviation fuel revealed the unsuitability of the silicone rubber diaphragm for prolonged usage in fuel [24]. A second set of sensors manufactured with a polyurethane based diaphragm showed no measurable deterioration over a 3-month period immersed in fuel. These sensors exhibited a sensitivity of $38.5 \pm 0.6 \mathrm{pm} / \mathrm{cm}$, which is less than the silicone rubber devices due to the stiffer nature of the polyurethane material used. Also, a study of the time-dependent variation of both the amplitude and resonance wavelength of four mPOFBG array sensors is presented and discussed when the sensors are in contact with fuel. In addition, a TOPAS mPOFBG was inscribed and embedded in polyurethane resin to analyze its behavior in contact with fuel. Our new system exhibits a high performance when compared with other previously published in the literature [25], making it a potentially useful tool for aircraft fuel monitoring.

\section{OPTICAL SENSOR FABRICATION}

For this study, a CW He-Cd laser (Kimmon IK3301R-G) with an output power of $30 \mathrm{~mW}$ at $325 \mathrm{~nm}$ and the phase mask technique have been used for the POFBG photo-inscription. All gratings were inscribed in a single-mode photosensitive PMMA fibre doped with benzyl dimethyl ketal [14]. Figure 1 (a) shows an optical microscope image of the cross section of the fiber. The average pitch $(\Lambda)$ and hole diameter $(d)$ are 4.26 and $1.87 \mu \mathrm{m}$, respectively, and the external diameter is $135 \mu \mathrm{m}$. This results in a relative hole diameter of $d /=0.44$; therefore, the fiber could have either a single or a few modes, depending on the wavelength. The final grating length was $2 \mathrm{~mm}$ obtained after 7 minutes of inscription. Two phase masks of $557.5 \mathrm{~nm}$ and $580 \mathrm{~nm}$ pitch were used in these experiments. Therefore, using PMMA fibres with a refractive index of 1.487 in that region, gratings can be formed with Bragg wavelengths centred approximately at $829 \mathrm{~nm}$ and $862 \mathrm{~nm}$, respectively. To change their wavelengths, thermal annealing of the inscribed fiber was used as discussed in [26]. Bragg grating wavelengths were monitored in reflection using a super-luminescent diode (Superlum SLD-371), an optical spectrum analyser (HP 86142A) and an $850 \mathrm{~nm}$ single-mode silica fiber coupler. Figure 1 (b) shows a typical reflection spectrum of a POFBGs array, measured by terminating one end of the mPOF with an $\mathrm{FC} / \mathrm{PC}$ connector [27]. After that, pressure sensing diaphragms were produced incorporating portions of fiber where each of the POFBGs were inscribed. A detailed discussion about diaphragm preparation can be found in [8,26]. Different linear POFBG arrays are embedded in two different types of diaphragms: silicone rubber (SILICAST from Dow Corning Corporation) and polyurethane resin (from Liquid Lens).

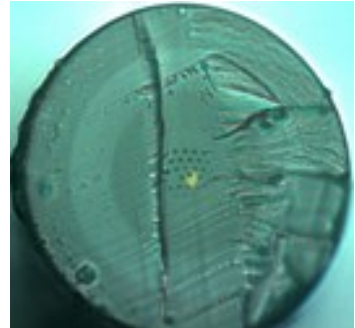

(a)

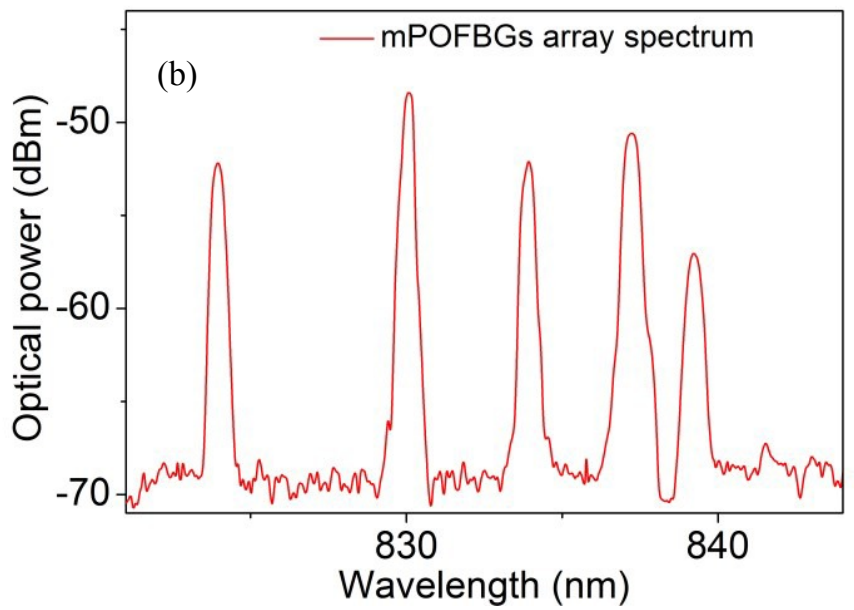

Figure 1. (a) Transversal section of the mPOF cut. (b) A typical mPOFBG array spectrum with five multiplexed gratings with their wavelengths changed by the thermal annealing process.

Five holes spatially separated by $15 \mathrm{~cm}$ were drilled on one side of a square acrylic tube $(800 \mathrm{~mm}$ length, with $3.2 \mathrm{~mm}$ wall thickness and $38.1 \mathrm{~mm}$ outside dimension). The sensors were then placed and sealed at positions aligned with the windows such that the FBG center was aligned with the window center. To fix the diaphragms in each position, retaining rings were used, with the diaphragm sandwiched between the tube and retaining ring. Eight screws were used to hold the 
tube and retaining ring together, producing a strong seal (see Figure 2 (a)). Figure 2 (b) shows the experimental apparatus and a diagram of the acrylic tube sensor arrangement using $\mathrm{mPOFBG}$ array sensors placed in contact with fuel.

(a)

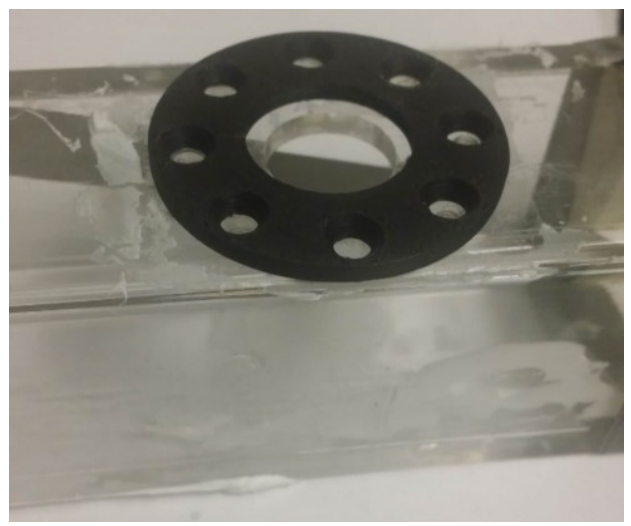

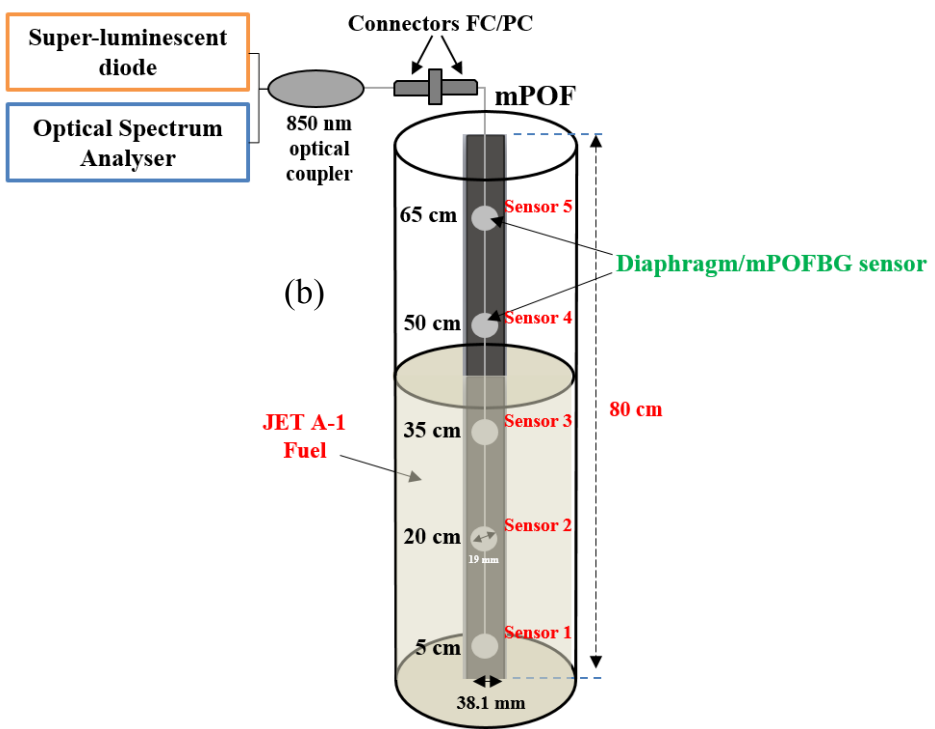

Figure 2. (a) Fixation of sensors to the square acrylic tube with retaining rings. (b) Experimental apparatus with three sensors submerged in fuel.

\section{RESULTS AND DISCUSSION}

Despite initially promising results with Jet A-1, the SILASTIC diaphragms were found to be unsuitable for long-term measurements as reported in [24]. After a few hours exposed to the fuel, the diaphragms were observed to swell to almost twice their original size as shown in Figure 3. Consequently a search was made for a more suitable material and a polyurethane resin from Liquid Lens [28] was chosen. This material is based on a mixture of two liquids - MF633 resin and DK780 catalyst - in a ratio of $100: 100$ by volume and curing at room temperature in 8 hours $\left(23^{\circ} \mathrm{C}, 40 \%\right.$ relative humidity). This material shows less flexibility than the previous one we used [24,26], however it is resistant to the fuel. A diaphragm with the same dimensions as the previous manufactured diaphragms was placed in JET A-1 for more than 3 months with no measurable change in size.

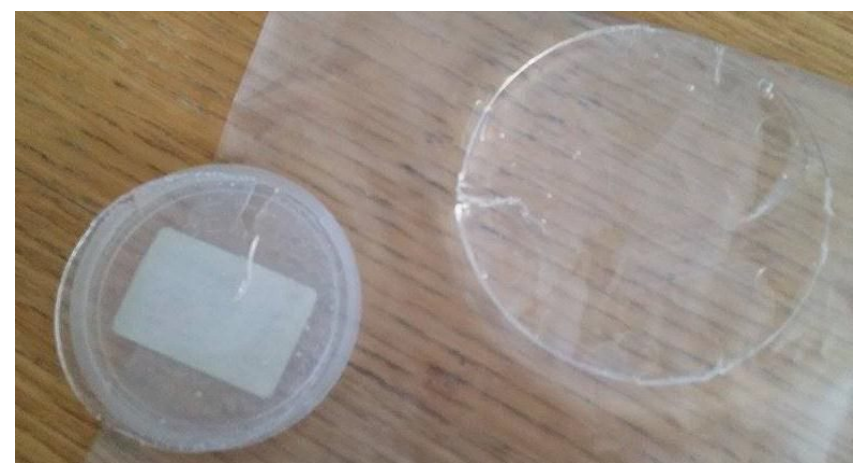

Figure 3. Diaphragms fabricated from SILASTIC silicone rubber. Left: as fabricated. Right: after exposure to fuel for several hours. 
The fuel level monitoring capability was tested within a liquid level range of 0 to $75 \mathrm{~cm}$ and with a liquid level increment step of $5 \mathrm{~cm}$. We conducted 2 series of experiments using different sensors. For each experiment, three cyclic tests were performed to investigate both increasing and decreasing levels of the liquid to check for any hysteresis in the behavior of the sensor. All cyclic tests showed a very good agreement and Figures 4 (a) and (b) show the first cycle of the first experiment for sensor 1 and sensor 3, with the position of each sensor indicated in Figure 2 (b). The wavelength shift was extracted and the sensitivity of each sensor (sensor 1,2,3,4 and 5) was calculated, showing an average sensitivity of $38.5 \pm 0.6 \mathrm{pm} / \mathrm{cm}$. The achieved sensitivity is less than with the SILASTIC silicone rubber $[8,26]$ due to the increased stiffness of the new material. The level resolution of sensor 1, as determined from the linear regression, was $1.0 \mathrm{~cm}$.
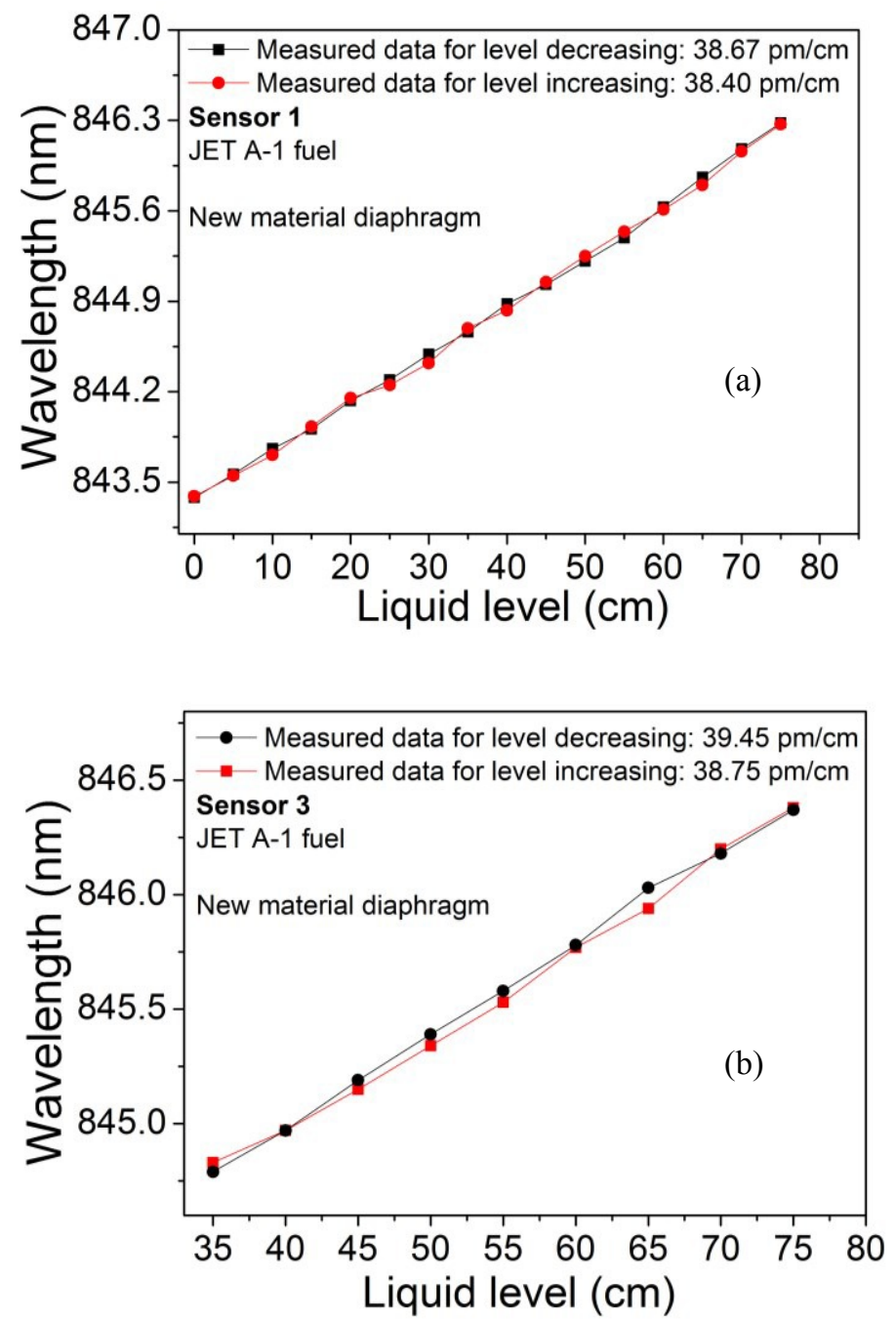

Figure 4. Cyclic response using the new diaphragm material (polyurethane resin from Liquid Lens [28]) in contact with JET A-1 for: (a) sensor 1 and (b) sensor 3.

In another experiment, the first three sensors (sensor 1, 2 and 3) were submerged to allow each to respond to pressure changes when the fuel is increased and decreased. The aim of this experiment was to study the behavior of three sensors submerged. Thus, the liquid depth was varied between $40 \mathrm{~cm}$ and $75 \mathrm{~cm}$, giving a $35 \mathrm{~cm}$ measurement region. Table 1 summarizes these results, showing the responses from sensor 1 , sensor 2 and sensor 3 and indicating a good agreement between their sensitivities. 
Table 1. Wavelength / sensitivity analysis of three submerged sensors.

\begin{tabular}{ccc}
\hline & $\begin{array}{c}\text { Increasing } \\
(\mathbf{p m} / \mathbf{c m})\end{array}$ & $\begin{array}{c}\text { Decreasing } \\
\text { (pm/cm) }\end{array}$ \\
\hline Sensor number & & \\
\hline Sensor 1 & $38.5 \pm 0.4$ & $38.8 \pm 0.3$ \\
Sensor 2 & $38.9 \pm 0.6$ & $38.4 \pm 0.5$ \\
Sensor 3 & $39.1 \pm 0.7$ & $38.5 \pm 1.0$ \\
\hline
\end{tabular}

In addition, experiments were carried out to evaluate the mPOFBG wavelength variation over time; both the reflectivity and the resonance wavelength shift were recorded over 90 days with a fuel level of $40 \mathrm{~cm}$. The experimental data were collected from sensors in contact with fuel. Room temperature was maintained at $23^{\circ} \mathrm{C}$ throughout the experiment. The behavior of sensor 2 is shown in Figure 5. From Figures 5 (a) and (b), we can observe that there is significant drift in the sensor response over a period of the first 70 days. The decrease of reflectivity after 90 days of experiment was $1.85 \mathrm{~dB}$ and the total wavelength shift was $-0.46 \mathrm{~nm}$ for this period, of which $-0.41 \mathrm{~nm}$ was the change resulting from the $20^{\text {th }}$ day to $90^{\text {th }}$ day. We speculate that the reason for the drift is due to the presence of water in the fuel, which is absorbed into the PMMA [29]. The reflectivity and wavelength variation values for the three sensors submerged in fuel were extracted and are listed in Table 2.
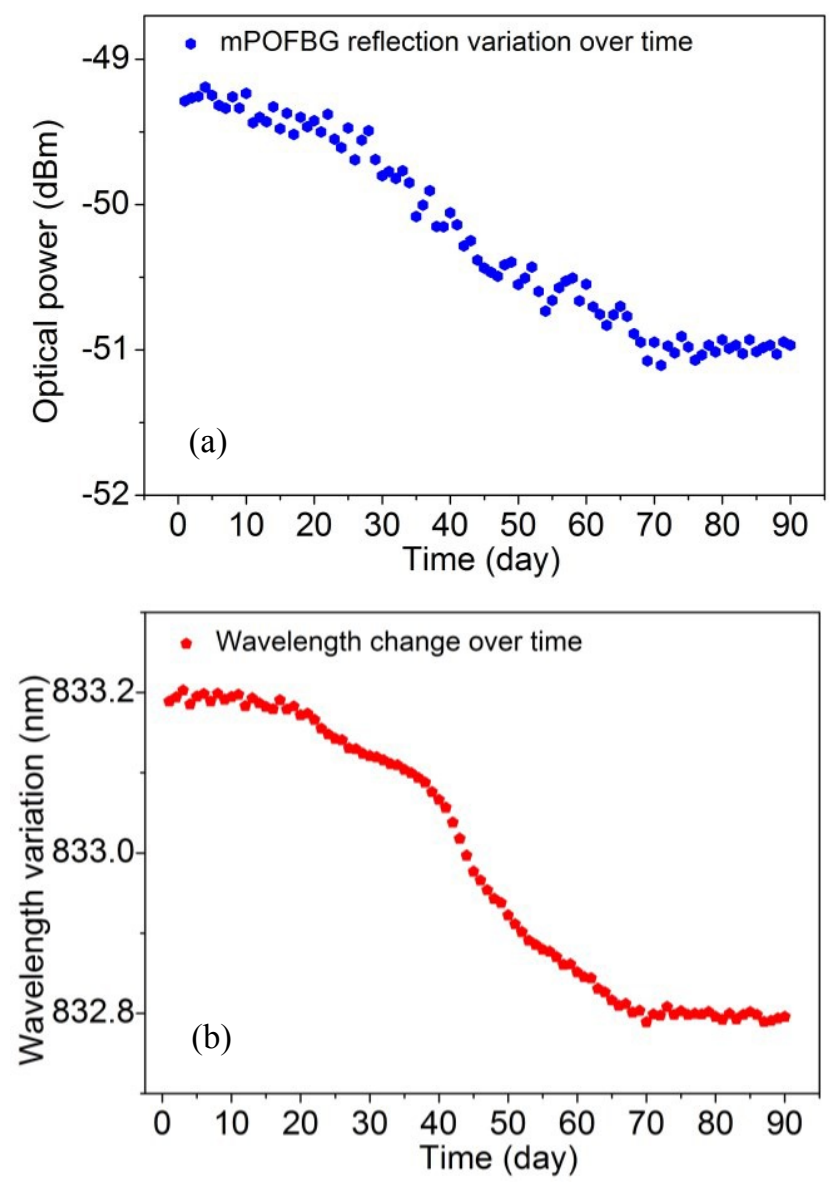

Figure 5. mPOFBGs reflection behavior (a) and resonance wavelength variation (b) over the time when the sensors are submerged in fuel. 
Table 2. Reflectivity and wavelength variation values for each sensor submerged in fuel.

\begin{tabular}{ccc}
\hline & $\begin{array}{c}\text { Reflectivity variation } \\
(\mathbf{d B})\end{array}$ & $\begin{array}{c}\text { Wavelength shift } \\
(\mathrm{nm})\end{array}$ \\
\hline Sensor number & & \\
\hline Sensor 1 & 1.92 & -0.50 \\
Sensor 2 & 1.85 & -0.46 \\
Sensor 3 & 2.08 & -0.54 \\
\hline
\end{tabular}

As a preliminary test, a TOPAS mPOFBG embedded in polyurethane resin was placed in contact with fuel to collect some measurements (reflectivity and wavelength changes) over 30 days. Figure 6 shows that there are no significant changes in the TOPAS mPOFBG optical spectrum since the TOPAS fiber is insensitive to humidity [15]. TOPAS fiber is a promising fiber type to use in this kind of applications where a significant quantity of water may be present.

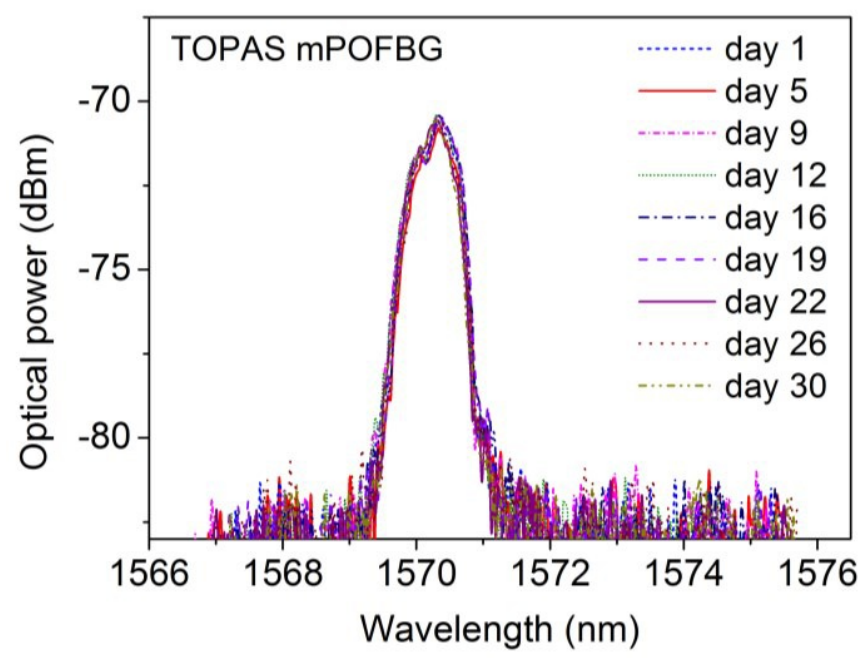

Figure 6. Reflected spectra of the TOPAS mPOFBG over 30 days when the sensor is submerged in fuel.

\section{CONCLUSION}

A fuel level sensor based on a single fiber, multiplexed array of mPOFBG sensors embedded in polyurethane resin diaphragms is designed and its performance studied for the first time. From these results, the proposed system using this type of diaphragm material shows good promise for prolonged operation in contact to fuel and the system has a good sensitivity to fuel level $(\sim 39 \mathrm{pm} / \mathrm{cm})$, presenting a highly linear and repeatable response. On the other hand, using silicone rubber diaphragms, this mPOFBG array sensor system exhibits a factor of 4 improvement in sensitivity $(\sim 98$ $\mathrm{pm} / \mathrm{cm}$ ) when compared with the best approach based on silica optical fiber published in the literature. However, despite the promising initial performance, longer term tests revealed the diaphragm material to be unsuitable for prolonged use [24]. In addition, tests in JET A-1 fuel to investigate the time-dependent variation of both the amplitude and resonance wavelength of mPOFBG array sensors embedded in diaphragms were reported. Long-term drifts were observed, possibly due to the sensitivity of PMMA to water. Initial tests with an alternative fiber material - TOPAS - revealed a much improved behavior, suggesting that TOPAS fiber should be further investigated for long-term fuel monitoring applications. 


\section{ACKNOWLEDGMENT}

This work was supported by Marie Curie Intra European Fellowships included in the 7th Framework Program of the European Union (projects PIEF-GA-2013-628604 and PIEF-GA-2011-302919). The research leading to these results has also received funding from the People Programme (Marie Curie Actions) of the European Union's 7th Framework Programme FP7/2007-2013/ under REA grant agreement No. 608382. The authors are grateful to M. Zubel by 3D printing of some plastic retaining rings used in this work.

\section{REFERENCES}

[1] Y. Shao, R. Cao, Y. K. Huang, P. N. Ji, S. Zhang, "112-Gb/s transmission over $100 \mathrm{~m}$ of graded-index POF for optical data center applications," Proc. Optical Fiber Communication Conference (OFC), OW3J.5 (2012).

[2] S. Zhou, L. Reekie, H. P. Chan, Y. T. Chow, P. S. Chung, K. M. Luk, "Characterization and modeling of Bragg gratings written in polymer fiber for use as filters in the THz region," Opt. Express 20, 9564 (2012).

[3] D. J. Webb, "Fiber Bragg grating sensors in polymer optical fibers," Meas. Sci. Technol. 26, 092004 (2015).

[4] K. Peters, "Polymer optical fiber sensors - A review," Smart Mater. Struct. 20, 013002 (2011).

[5] S. Kiesel, P. Van Vickle, K. Peters, T. Hassan, M. Kowalsky, "Intrinsic polymer optical fiber sensors for highstrain applications," Proc. SPIE 6167, Smart Structures and Materials, 616713-11 (2006).

[6] C. A. F. Marques, L. Bilro, L. Kahn, R. A. Oliveira, D. J. Webb, R. N. Nogueira, "Acousto-Optic effect in microstructured polymer fiber Bragg gratings: simulation and experimental overview", IEEE/OSA J. Lightw. Technol. 31, 1551 (2013).

[7] T. X. Wang, Y. H. Luo, G. D. Peng, Q. Zhang, "High-sensitivity stress sensor based on Bragg grating in BDKdoped photosensitive polymer optical fiber", Proc. 3rd Asia Pacific Optical Sensors Conference, 83510M (2012).

[8] C. A. F. Marques, G. D. Peng, David J. Webb, "Highly sensitive liquid level monitoring system utilizing polymer fiber Bragg gratings," Opt. Express 23, 6058 (2015).

[9] H. B. Liu, H. Y. Liu, G. D. Peng, P. L. Chu, "Strain and temperature sensor using a combination of polymer and silica fibre Bragg gratings," Opt. Commun. 219, 139 (2003).

[10] X. S. Cheng, W. W. Qiu, W. X. Wu, Y. Luo, X. Tian, Q. Zhang, B. Zhu "High-sensitivity temperature sensor based on Bragg grating in BDK-doped photosensitive polymer optical fiber," Chinese Opt. Letters 9, 020602 (2011).

[11] W. Zhang, D. J. Webb, G. D. Peng, "Investigation into time response of polymer fiber Bragg grating based humidity sensors," IEEE/OSA J. Lightw. Technol. 30, 1090 (2012).

[12] X. Hu, D. Sáez-Rodríguez, C. A. F. Marques, O. Bang, D. J. Webb, P. Mégret, C. Caucheteur, "Polarization effects in polymer FBGs: study and use for transverse force sensing," Opt. Express 23, 4581 (2015).

[13] C. A. F. Marques, L. Bilro, N. J. Alberto, D. J. Webb, R. N. Nogueira, "Narrow bandwidth Bragg gratings imprinted in polymer optical fibers for different spectral windows," Opt. Commun. 307, 57 (2013).

[14] D. Sáez-Rodríguez, K. Nielsen, H. K. Rasmussen, O. Bang, D. J. Webb, "Highly photosensitive polymethyl methacrylate microstructured polymer optical fiber with doped core," Opt. Letters 38, 3769 (2013).

[15] W. Yuan, L. Khan, D. J. Webb, K. Kalli, H. K. Rasmussen, A. Stefani, O. Bang, "Humidity insensitive TOPAS polymer fiber Bragg grating sensor," Opt. Express 19, 19731 (2011).

[16] A. Lacraz, M. Polis, A. Theodosiou, C. Koutsides, K. Kalli, "Femtosecond laser inscribed Bragg gratings in low loss CYTOP polymer optical fibre" IEEE Phot. Techn. Letters 27, 693 (2015).

[17] R. Oliveira, C. A. F. Marques, L. Bilro, R. N. Nogueira, "Production and characterization of Bragg gratings in polymer optical fibers for sensors and optical communications," Proc. 23rd International Conference on Optical Fibre Sensors - OFS 23, 915794 (2014).

[18] R. Oliveira, L. Bilro, R. N. Nogueira, "Bragg gratings in a few mode microstructured polymer optical fiber in less than 30 seconds," Opt. Express 23, 10181 (2015).

[19] J. D. Weiss, "Fluorescent optical liquid-level sensor", Opt. Eng. 39, 2198 (2000).

[20] B. Yun, N. Chen, Y. Cui, "Highly sensitive liquid-level sensor based on etched fiber Bragg grating," IEEE Phot. Techn. Letters 19, 1747 (2007).

[21] B. Gu, W. Qi, Y. Zhou, Z. Wu, P. P. Shum, F. Luan, "Reflective liquid level sensor based on modes conversion in thin-core fiber incorporating titled fiber Bragg grating," Opt. Express 22, 11834 (2014).

[22] A. L. Ricchiuti, D. Barrera, K. Nonaka, S. Sales, "Fiber optic liquid-level sensor using a long fiber Bragg grating," Proc. 5th European Workshop on Optical Fibre Sensors, 87941J (2013). 
[23] D. Sengupta, P. Kishore, “Continuous liquid level monitoring sensor system using fiber Bragg grating,” Opt. Eng. 53, 017102 (2014).

[24] C. A. F. Marques, A. Pospori, D. Sáez-Rodríguez, K. Nielsen, O. Bang, D. J. Webb, "Aviation fuel gauging sensor utilizing multiple diaphragm sensors incorporating polymer optical fiber Bragg gratings," submitted to IEEE/OSA J. Lightw. Technol. (2016).

[25] C. Zhao, L. Ye, X. Yu, J. Ge, "Continuous Fuel Level Sensor Based on Spiral Side-Emitting Optical Fiber," Journal of Control Science and Engineering 2012, (2012).

[26] C. A. F. Marques, A. Pospori, D. Sáez-Rodríguez, K. Nielsen, O. Bang, D. J. Webb, "Fiber-optic liquid level monitoring system using microstructured polymer fiber Bragg grating array sensors: performance analysis", Proc. 24th International Conference on Optical Fibre Sensors - OFS 24, 9634-106 (2015).

[27] A. Abang, D. Sáez-Rodríguez, K. Nielsen, O. Bang, D. J. Webb, "Connectorisation of fibre Bragg grating sensors recorded in microstructured polymer optical fibre", Proc. 5th European Workshop on Optical Fibre Sensors, 87943Q (2013).

[28] http://www.liquid-lens.com/advanced2 resin.html

[29] W. Zhang, D. J. Webb, M. Carpenter, C. Williams, "Measuring Water Activity of Aviation Fuel Using a Polymer Optical Fiber Bragg Grating", Proc. 23rd International Conference on Optical Fibre Sensors - OFS23, 91574V-1 (2014). 